\title{
Prevention of NSAID-Enteropathy: A Soluble Problem?
}

\author{
John L. Wallace ${ }^{1}$
}

Published online: 17 November 2015

(c) Springer Science+Business Media New York 2015

Small intestinal injury caused by nonsteroidal anti-inflammatory drugs (NSAIDs), common and clinically significant [1], is difficult to detect and largely asymptomatic, with associated hospital costs and rates of mortality higher than those for NSAID-induced upper gastrointestinal (GI) injury [2]. There are no proven-effective preventative or therapeutic options available to physicians [3]. Indeed, in animal studies, anti-secretory drugs that are commonly used to reduce the incidence of NSAID-induced damage in the upper GI tract [proton pump inhibitors (PPI), histamine $\mathrm{H}_{2^{-}}$ receptor antagonists $\left.\left(\mathrm{H}_{2} \mathrm{RA}\right)\right]$ markedly exacerbate NSAID-associated jejunal and ileal damage [4, 5], in part through significant changes to the intestinal microbiota that include a marked reduction in numbers of Bifidobacteria [5], and by increasing the cytotoxicity of bile [6]. A recent capsule endoscopic study of NSAID-enteropathy in patients with arthritis identified the three strongest risk factors for developing severe intestinal damage and reduced hematocrit: use of a PPI, use of an $\mathrm{H}_{2} \mathrm{RA}$, and advanced age (odds ratios of 5.2, 4.0, and 4.2, respectively; all $p<0.05)$ [7].

Rodent models have been very useful for studying the pathogenesis of this condition, since the factors contributing to the damage in such models appear to be similar to those in humans: alterations in the intestinal microbiota, enterohepatic circulation of the NSAID, increased cytotoxicity of bile, and other factors [3] (Fig. 1). Although altering the intestinal microbiota with antibiotics and/or

John L. Wallace

altapharm@hotmail.com

1 Department of Physiology and Pharmacology, University of Calgary, 3330 Hospital Drive NW, Calgary, AB T2N 4N1, Canada reducing the enterohepatic circulation of the NSAID all markedly diminish NSAID-enteropathy in rodents [3], some of these approaches are not viable, nor desirable, therapeutic strategies for humans, since they can also reduce the therapeutic efficacy of the NSAID. Animal studies of prophylaxis with antibiotics have yielded inconsistent or conflicting results $[8,9]$, further complicated by concern about the development of resistant strains of bacteria, particularly if long-term or repeated use of antibiotics is required for effectiveness [10].

In this issue of Digestive Diseases and Sciences, Satoh et al. [11] report that NSAID-induced intestinal damage in arthritic rats could be markedly reduced or prevented simply through the provision of a diet enriched in soluble fiber. After arthritis had been induced and established in rats by injection of Freund's adjuvant, the rats were placed on a powdered diet alone, or one supplemented with one of two soluble fibers: pectin or guar gum. Two days later, a 3-day course of indomethacin treatment was started, with blind evaluation of the intestinal damage occurring 1 day after the final treatment. The authors observed a 60-90\% reduction in intestinal damage in the rats receiving the pectin- or guar gum-supplemented diets as compared to controls. Importantly, the dietary interventions did not attenuate the desired anti-inflammatory effects of the NSAID. Also, feeding the rats an enriched with insoluble (cellulose-based) dietary fiber diet did not significantly alter the severity of NSAID-induced intestinal damage [11].

There are several potential mechanisms of action that could be invoked to explain the remarkable effectiveness of the diets high in soluble fiber in this rat model. A favorable alteration in the intestinal microbiome would be logical candidate. NSAID administration to rodents substantially alters the numbers and types of bacteria in the intestine; in 


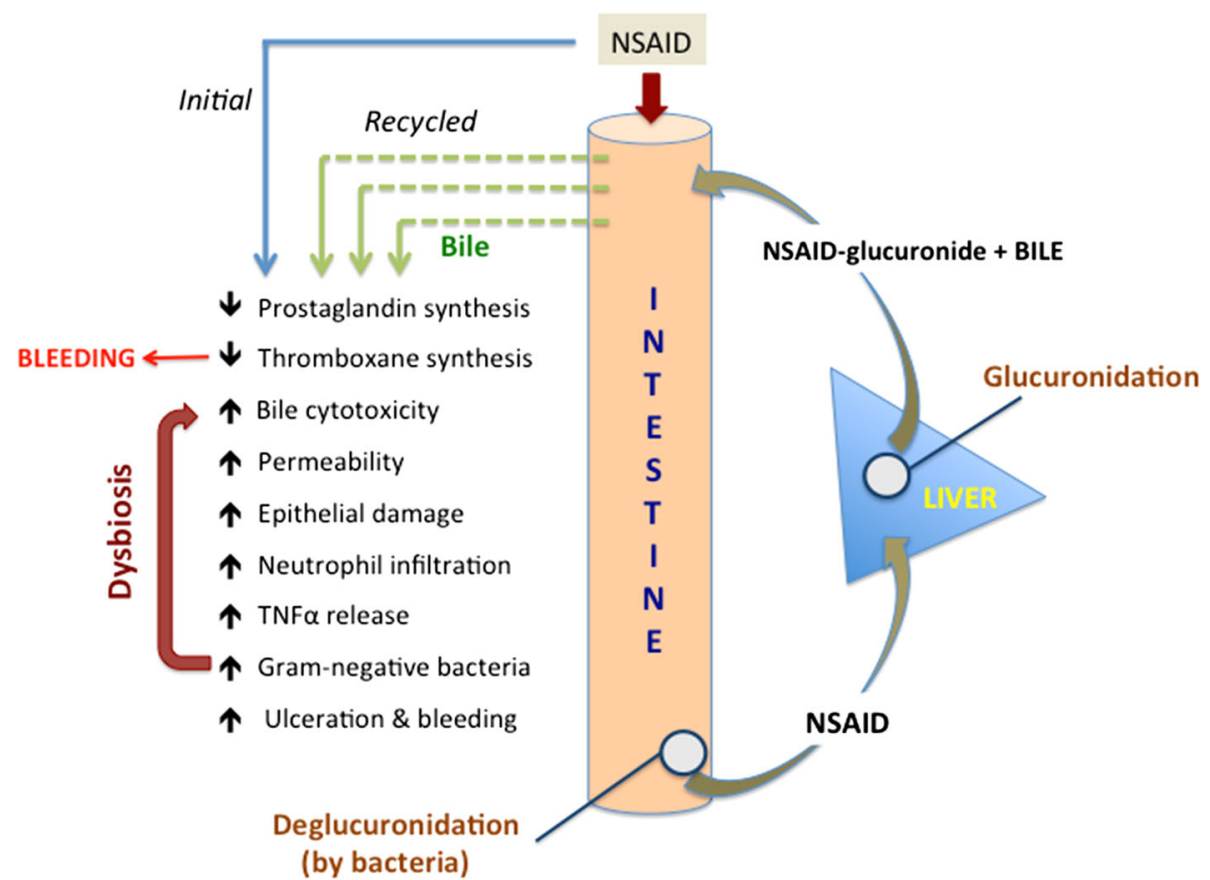

Fig. 1 Pathogenesis of NSAID-enteropathy: Multiple mechanisms contribute to the small intestinal damage caused by nonsteroidal antiinflammatory drugs (NSAIDs). All NSAIDs suppress cyclooxygenase activity, thereby decreasing synthesis of prostaglandins (which are protective in the gastrointestinal tract) and thromboxane (which promotes blood clotting). Bile, enteric bacteria, and the enterohepatic recirculation of NSAIDs are all important factors in the production of intestinal injury, and they are also interrelated, since bile can affect the composition of the intestinal microbiota, and the microbiota can alter the metabolism, absorption, and cytotoxicity of bile. NSAIDs

general terms, there is a marked increase in numbers of Gram-negative bacteria (which tend to promote tissue injury, in part through release of endotoxin) and a significant decrease in Gram-positive bacteria (many of which exert protective effects in the GI tract) $[3,4,8]$. Diets enriched in pectin or guar gum produce health-benefitting modifications to the rat microbiome [12, 13]. For example, pectin decreases cecal colonization with the Gram-negative bacterium Bacteroidetes, while increasing colonization with Gram-positive Firmicutes [12]. Guar gum diets similarly increase intestinal colonization with Bifidobacteria [13], the same group of bacteria that was significantly diminished in rats treated with PPIs, and associated with increased severity of NSAID-enteropathy [4]. Importantly, pectin and guar gum diets promote the growth of bacteria that produce butyrate $[12,13]$, a short-chain fatty acid that is important for the maintenance of epithelial barrier integrity. Short-chain fatty acids can trigger release of glucagon-like peptide-2 from L-cells in the intestine and therefore promote healing of tissue injury (such as that induced by NSAIDs) via stimulation of epithelial proliferation $[14,15]$. There is good evidence that certain probiotics, including Bifidobacteria ssp., Lactobacillus ssp., significantly change the composition of the intestinal microbiota (dysbiosis), with a loss of Gram-positive bacteria and a marked increase in Gram-negative bacteria; such changes are associated with reduced mucosal defense and an increase susceptibility to damage. NSAID-induced dysbiosis is also associated with a marked increase in the cytotoxicity of bile on epithelial cells. Endotoxin release from Gram-negative bacteria further exacerbates mucosal injury, in part through induction of release of pro-inflammatory cytokines such as tumor necrosis factor- $\alpha$

and Faecalbacterium prausnitizii, can exert beneficial effects against NSAID-enteropathy in animal models [8, 9]. Diets enriched with soluble fiber can also modulate bile flow and bind certain bile acids, which could further contribute to reducing the severity of NSAID-induced enteropathy [16].

Could a dietary approach to prevention of NSAID-enteropathy be successful in humans? If it is assumed that a diet enriched with soluble fiber would produce a similar effect in humans as was observed in rats, then such a diet should be a relatively inexpensive preventative strategy, offering the additional well-documented health benefits of a higher-fiber diet [17]. This approach would be attractive to some patients, assuming that, as in the rats, there is no diminution of the efficacy of anti-inflammatory therapy for the patient. There is potential for very good compliance, particularly in patients who are seeking "natural" rather than drug-based remedies. On the other hand, since compliance is often a limiting factor with dietary approaches to disease management (e.g., obesity, type 2 diabetes, hypertension), particularly if the patient does not perceive a clear benefit of the strategy, a dietary approach could be a significant issue for prevention of NSAID-enteropathy. Taking an inhibitor of gastric acid 
secretion to relieve the symptoms of acid reflux works well, in part because the patient experiences a significant "signal" (pain), and that signal is attenuated after taking the antisecretory drug. However, NSAID-induced enteropathy is largely an asymptomatic disorder, with no patient-perceived signal to trigger and maintain compliance with a treatment regimen. If there were a reliable biomarker of NSAIDenteropathy, it could be used as the positive feedback for the patient to continue with a dietary regimen, in much the same way that regular measurement of blood cholesterol is an encouragement for patients to continue taking a lipid-lowering statin. At present, the noninvasive biomarkers for NSAID-induced damage are limited: examples include monitoring changes in hematocrit, fecal blood, or fecal calprotectin.

There may be significant patient-to-patient variability in the amount of soluble dietary fiber required to produce beneficial effects, and it is possible that some patients will not tolerate the diet. Whether or not the short-term benefits demonstrated in the rat study can be maintained over the many years that patients with diseases like arthritis take NSAIDs is another uncertainty.

An alternative approach to prevention of NSAID-enteropathy that would be simpler in application and more easily controlled in a clinical setting would be the use of specific probiotics. The main advantages of this strategy, assuming that it is effective, would be greater patient compliance. A suitable probiotic or combination of probiotics may be sufficient to attenuate or reverse the intestinal dysbiosis caused by NSAIDs and therefore prevent intestinal damage. The primary challenge for this approach will be the formulation of an appropriate number and mix of organisms into a tablet. Given the substantial economic costs and morbidity associated with NSAID-enteropathy, and the continued growth in the use of this class of drugs, a strong case can be made for taking on such a challenge.

\section{Compliance with ethical standards}

Conflict of interest Dr. Wallace is the founder and a director of Antibe Therapeutics Inc.

\section{References}

1. Lanas A, Sopena F. Nonsteroidal anti-inflammatory drugs and lower gastrointestinal complications. Gastroenterol Clin North Am. 2009;38:333-352.
2. Lanas A, Garcia-Rodriguez LA, Polo-Tomas M, et al. Time trends and impact of upper and lower gastrointestinal bleeding and perforation in clinical practice. Am $J$ Gastroenterol. 2009;104:1633-1641.

3. Wallace JL. Mechanisms, prevention and clinical implications of nonsteroidal anti-inflammatory drug-enteropathy. World J Gastroenterol. 2013;19:1861-1876.

4. Wallace JL, Syer S, Denou E, et al. Proton pump inhibitors exacerbate NSAID-induced small intestinal injury by inducing dysbiosis. Gastroenterology. 2011;141:1314-1322.

5. Satoh H, Amagase K, Takeuchi K. Exacerbation of nonsteroidal anti-inflammatory drug-induced small intestinal lesions by antisecretory drugs in rats: the role of intestinal motility. $J$ Pharmacol Exp Ther. 2012;343:270-277.

6. Blackler RW, De Palma G, Manko A, et al. Deciphering the pathogenesis of NSAID enteropathy using proton pump inhibitors and a hydrogen sulfide-releasing NSAID. Am J Physiol Gastrointest Liver Physiol. 2015;308:G994-G1003.

7. Watanabe T, Tanigawa T, Nadatani $\mathrm{Y}$, et al. Risk factors for severe nonsteroidal anti-inflammatory drug-induced small intestinal damage. Dig Liver Dis. 2013;45:390-395.

8. Watanabe T, Nishio H, Tanigawa T, et al. Probiotic Lactobacillus casei strain Shirota prevents indomethacin-induced small intestinal injury: involvement of lactic acid. Am J Physiol Gastrointest Liver Physiol. 2009;297:G506-G513.

9. Syer SD, Blackler RW, Martin R, et al. NSAID enteropathy and bacteria: a complicated relationship. J Gastroenterol. 2015; 50:387-393.

10. Lanas A, Scarpignato C. Microbial flora in NSAID-induced intestinal damage: a role for antibiotics? Digestion. 2006; 73:136-150.

11. Satoh H, Matsumoto H, Hirakawa T, Wada N. Soluble dietary fibers can protect the small intestinal mucosa without affecting the anti-inflammatory effect of indomethacin in adjuvant-induced arthritis rats. Dig Dis Sci. (Epub ahead of print). doi:10.1007/ s10620-015-3889-0.

12. Licht TR, Hansen M, Bergstrom A, et al. Effects of apples and specific apple components on the cecal environment of conventional rats: role of apple pectin. BMC Microbiol. 2010;10:13.

13. Ohashi Y, Sumitani K, Tokunaga M, Ishihara N, Okubo T, Fujisawa T. Consumption of partially hydrolysed guar gum stimulates Bifidobacteria and butyrate-producing bacteria in the human large intestine. Benef Microbes. 2015;6:451-455.

14. Akiba Y, Inoue T, Kaji I, et al. Short-chain fatty acid sensing in rat duodenum. J Physiol. 2015;593:585-599.

15. Inoue T, Higashiyama M, Kaji I, et al. Dipeptidyl peptidase IV inhibition prevents the formation and promotes the healing of indomethacin-induced intestinal ulcers in rats. Dig Dis Sci. 2014;59:1286-1295.

16. Kritchevsky D. Influence of dietary fiber on bile acid metabolism. Lipids. 1978;13:982-985.

17. Roberfroid M, Gibson GR, Hoyles L, et al. Prebiotic effects: metabolic and health benefits. Br J Nutr. 2010;104:S1-S63. 